\title{
Infarto agudo de miocardio como manifestación inicial de síndrome antifosfolípido primario: Reporte de Caso
}

\author{
Laura Camila Pedraza Areváloํ, Renato Antonio Guzmán Moreno²*
}

\begin{abstract}
${ }^{1}$ Médico Interno Investigación, Facultad de Medicina, Fundación Universitaria Juan N. Corpas, Bogotá D.C., Bogotá. (Colombia). ${ }^{2}$ Médico Internista, Especialista en Inmunología y Reumatología, Instituto de Enfermedades Autoinmunes Renato Guzmán, Bogotá D.C., Colombia. Profesor Titular de Inmunología y Medicina Interna. Departamento de Medicina Interna, Fundación Universitaria Juan N. Corpas, Clínica Corpas. Bogotá. (Colombia).
\end{abstract}

\section{RESUMEN}

Introducción. El síndrome antifosfolípido primario (SAF) suele manifestarse con trombosis venosas más que con arteriales, y entre estas últimas las comunes son a nivel cerebral, sin embargo, también pueden debutar con infarto agudo de miocardio (IAM). Por ello, es importante conocer el contexto en el cual sospechar eventos trombóticos como manifestación de SAF, en especial en el corazón dado que impactan en el pronóstico. El presente trabajo describe un caso de síndrome SAF en un hombre de 29 años que acude a urgencias con dolor en miembro inferior izquierdo asociado a signos de inflamación local que impiden la marcha, antecedente de SAF dado por 2 IAM y una trombosis venosa profunda (TVP) por lo cual estaba anticoagulado con cumarínico. El examen físico fue compatible con TVP izquierda, que luego se confirmó con Doppler, por lo que se considera una recurrencia de la enfermedad de base y se inicia manejo con heparina de bajo peso molecular; el paciente tiene una mejoría clínica al 5 día de hospitalización por lo que se da alta hospitalaria. A continuación, se discute el IAM como la primera manifestación de SAF, resaltando sus causas, presentación, manejo y pronóstico.

Palabras clave: Síndrome antifosfolípido primario, Manifestación, Infarto agudo de miocardio.

\section{ABSTRACT}

\section{Acute myocardial infarction as initial manifestation of primary antiphospholipid syndrome: Case Report}

Introduction. Primary antiphospholipid syndrome (APS) is commonly manifested with venous thrombosis more than with arterial thrombosis. In the latter, the most common thrombosis is cerebral, although it can debut with acute myocardial infarction (AMI). According to that, is important to know the context in which we must suspect thrombotic events as APS manifestation, especially in the heart taking into account the impact in the prognosis. This paper describes a case of APS in a 29-years-old male who was admitted in the emergency room

with pain in the left lower limb associated to signs of local
\end{abstract}

inflammation that blocks walking; history of primary APS due to $2 \mathrm{AMI}$ and one deep venous thrombosis (DVT) whereby the patient was anticoagulated with coumarin. The physical exam was compatible with left DVT, that was confirmed after with Doppler, so there is considered a recurrence of the base disease and low molecular weight heparin was initiated. At fifth day of hospitalization, the patient had clinical improvement, so he was discharged. Next, AMI is discussed as first manifestation of APS, highlighting the causes, presentation, management and prognosis.

Keywords: Primary antiphospholipid syndrome, Manifestation, Acute myocardial infarction.

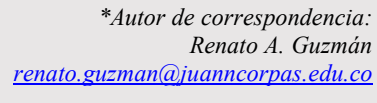

Como citar: Pedraza Arévalo, LC, Guzmán Moreno, RA, Infarto agudo de miocardio como manifestación inicial de sindrome antifosfolipido primario: Reporte de Caso. Revista Cuarzo 2019;25(2):38-41.

Recibido: 23 de julio de 2019 Aceptado: 25 de septiembre de 2019 Publicado: 30 de diciembre de 2019 DOI: https://doi.org/10.26752/cuarzo.v25.n2.444 


\section{INTRODUCCIÓN}

$\mathrm{E}$ $1 \mathrm{SAF}$ es una patología que se distingue por presentarse entre los 15 y los 50 años con manifestaciones trombofílicas tanto arteriales como venosas, con una relación mujer: hombre de 3.1:1. La presentación más frecuente es la TVP $(1,2)$, sin embargo, las trombosis arteriales pueden ocurrir hasta en el $33 \%$ de los pacientes con SAF (3), con el IAM como la primera manifestación de un SAF primario en un $1.2 \%$ de los pacientes, incluso en menos del $1 \%$ en la cohorte de Davies et al., alcanzando una mortalidad del 10\% (4-7). Las manifestaciones cardiovasculares del SAF son diversas y se han descrito, entre otras, la enfermedad coronaria, angor pectoris, valvulopatías, miocardiopatías, vegetaciones, trombosis intracardiaca, hipertensión pulmonar, disfunción miocárdica y TEP (7-9).

Los mecanismos que pueden generar IAM en este contexto son: la ateroesclerosis acelerada por la interacción entre los anticuerpos antifosfolípido y el colesterol de baja densidad, la injuria microvascular y el tromboembolismo coronario, siendo este último más frecuente en pacientes jóvenes $(7,10)$.

Por otra parte, es llamativo que se han encontrado las arterias coronarias normales en el $75 \%$ de pacientes con SAF que debutan con IAM (4), sugiriendo que los mecanismos son diferentes en frecuencia comparados con la población en general, atribuyéndolo entonces a trombosis coronaria, embolismos o IAM con coronarias no obstructivas, más conocido como MINOCA por sus siglas en inglés. Así lo reporto un estudio con 545 pacientes con anticuerpos antifosfolípidos presentes en suero, de los cuales 40 fueron a cateterismo cardiaco y 8 tenían hallazgos de $\operatorname{MINOCA}(6,11)$.

En general, el abordaje terapéutico es similar a los casos de IAM sin SAF y los resultados son satisfactorios, pero debe recordarse que en estos casos hay una patología subyacente de base, de naturaleza trombótica y recurrente y que la anticoagulación hace parte de su manejo esencial para el resto de sus vidas (12-16).

Teniendo en cuenta que el SAF puede producir IAM e impacta en el pronóstico de este grupo de pacientes, se presenta un caso clínico de SAF primario en paciente joven con trombosis recurrentes, entre ellas, IAM.

\section{PRESENTACIÓN DEL CASO}

\section{Información del paciente.}

Paciente masculino de 29 años, natural de Bogotá y residente de Zipaquirá, quien acude al servicio de urgencias por cuadro clínico de 3 días de evolución, consistente en dolor en miembro inferior izquierdo asociado a signos de inflamación local como cambios en la coloración de la piel de rojo a violáceo, edema y calor progresivos que impiden la marcha. El paciente se automedicó con acetaminofén sin mejoría de los síntomas. Como antecedentes personales refiere SAF desde hace 10 años, cuyo diagnóstico se realizó por presentar un IAM con necesidad de revascularización, asociado a anticuerpos antifosfolípidopositivos en una primera ocasión y hace 7 años con un segundo IAM, además de TVP derecha hace 6 meses, por lo que estaba anticoagulado con Warfarina $10 \mathrm{mg}$ los días domingo, lunes, miércoles y jueves, y $5 \mathrm{mg}$ resto de días.

\section{Hallazgos clínicos.}

$\mathrm{Al}$ examen físico, los signos vitales se encontraban dentro de la normalidad; en las extremidades se evidenció mayor diámetro del miembro inferior izquierdo versus el derecho $(46 \mathrm{~cm}$ a nivel de gastrocnemios y $22 \mathrm{~cm}$ a nivel del tobillo versus $31 \mathrm{~cm}$ a nivel de gastrocnemios y $20 \mathrm{~cm}$ a nivel del tobillo, respectivamente) con signos de Homans y Pratt positivos. El resto del examen físico era normal.

\section{Diagnóstico y tratamiento.}

Se considera entonces, diagnósticos de TVP izquierda y SAF; se hospitaliza por el servicio de medicina interna, se anticoagula con heparina de bajo peso molecular $1 \mathrm{mg} / \mathrm{kg}$ cada 12 horas y se solicita Doppler venoso de miembro inferior izquierdo, cuadro hemático, tiempos de coagulación e interconsulta a reumatología. Se recibe reporte de Doppler que indica trombosis aguda íleo-femoro-poplítea y edema a lo largo de todo el miembro inferior; cuadro hemático normal y tiempos de coagulación prolongados. Al segundo día de hospitalización el paciente es valorado por el servicio de reumatología, que considera que efectivamente cumple criterios para SAF y que se debe continuar el manejo instaurado, adicionando la solicitud de perfil autoinmune para definir si es primario o secundario, recibiendo resultados de ENAs y ANAs negativos, estos últimos con título de 1/80 patrón MSA2, descartando así clínica y serológicamente un lupus eritematoso sistémico de base; anticoagulante lúpico y anticuerpos anti cardiolipinas $\operatorname{IgM}$ e IgG positivos, ratificando el diagnóstico de SAF primario. Se continua con el manejo instaurado, con una mejoría del dolor y disminución del edema al 4 día de hospitalización y mejoría de la movilización el 5 día, siendo posible el alta hospitalaria con heparina de bajo peso molecular $1 \mathrm{mg} / \mathrm{kg}$ cada 12 horas durante 30 días, cita de control por medicina interna y reumatología. En el control ambulatorio el paciente estaba asintomático, manejado con Warfarina $10 \mathrm{mg}$ al día y controles de tiempos de coagulación. Se sugirió valoración por el servicio de hematología. cinco meses después de la última TVP, se hizo contacto telefónico con el paciente, quien comento que no ha tenido nuevos episodios trombóticos desde entonces, sin embargo, por barreras de acceso al servicio de salud, tampoco ha vuelto a estar en control ambulatorio. En la Tabla 1 se resumen los acontecimientos relevantes del caso en mención.

Tabla 1: Acontecimientos Relevantes

\begin{aligned} \hline \multicolumn{1}{c}{ Tiempo } & \multicolumn{1}{c}{ Evento } \\ \hline \hline 10 años atrás & $\begin{array}{l}\text { Infarto agudo de miocardio como manifestación } \\ \text { inicial de síndrome antifosfolípido }\end{array} \\ 7$ años atrás & Segundo infarto agudo de miocardio \\ Hace 6 meses & Trombosis venosa profunda derecha \\ Ahora & Trombosis venosa profunda izquierda \\ \hline \hline & Fuente: Descripción realizada por los Autores \end{aligned}




\section{DISCUSIÓN}

Cuando estamos ante un paciente joven y sin factores de riesgo para enfermedad cardiovascular, que presenta IAM, vale la pena descartar un SAF (17), si bien la trombosis arterial cerebral es más común que la coronaria en estos pacientes, pues se ha reportado que en el $13 \%$ y $11 \%$ respectivamente, se encuentran anticuerpos antifosfolípido positivos (18). El MINOCA es cada vez más frecuentemente encontrado en pacientes con IAM y condiciones trombofílicas que pueden explicar el aumento de su prevalencia, pues los fenómenos trombóticos pueden generar vasoespasmo que conllevan a síndromes coronarios siendo cada vez más asociados con SAF primario. Un estudio encontró que, de 84 pacientes con MINOCA y 84 con evento cerebrovascular criptogénico, $15.5 \%$ y $10 \%$ respectivamente tenían los 3 anticuerpos antifosfolípidopositivos, comparado con el $1-5 \%$ de la población general. También reportaron que, de 40 pacientes con IAM con elevación del ST y 44 sin elevación del ST, $2.5 \%$ y $27.3 \%$ respectivamente tenían los 3 anticuerpos antifosfolípido positivos (19).

En el caso expuesto, un paciente joven con trombosis a recurrencia que se presentó con TVP izquierda, evolucionó satisfactoriamente con el tratamiento anticoagulante, pero con un importante antecedente de 2 IAM, confirmando lo que se reporta en la literatura al respecto, pues se ha visto que las recurrencias y trombosis de stent son más comunes en pacientes con SAF al compararlos con la población general sometida a Intervención Coronaria Percutánea y con pacientes con IAM con elevación del ST sin SAF, conllevando a un incremento en la tasa de eventos adversos cardiovasculares mayores (20). Así pues, estos pacientes suelen ser revascularizados, principalmente por reestenosis y por la ateroesclerosis acelerada en vasos no cubiertos por el stent, resaltando que los anticuerpos anticardiolipinas elevados son un factor predictor de reestenosis (20). Otro aspecto del manejo de estos pacientes es la anticoagulación, que debe ser de por vida y el medicamento recomendado es la Warfarina, con niveles exigentes de INR, mayores de 3, o INR entre 2 y 3 adicionando aspirina a bajas dosis (21). En el caso expuesto, el paciente ingreso con un INR de 1.3, fuera del rango terapéutico, lo que puede explicar la actividad de la enfermedad. Intrínsecamente, en el contexto de SAF primario el riesgo cardiovascular es elevado, dado principalmente por una etiopatogenia proinflamatoria secundaria a activación de monocitos, células endoteliales, plaquetas, complemento e interferencia con las proteínas involucradas en la cascada de la coagulación $(20,22,23)$ por lo que, cuando el paciente debuta con compromiso cardiaco, no solo la anticoagulación sino también las estatinas y la hidroxicloroquina pueden ser beneficiosos en este escenario $(24,25)$.

Se ha tratado de asociar esta complicación al subtipo del anticuerpo, sin embargo, la evidencia no es concluyente, pues en algunos casos se ha relacionado más con el anticuerpo antibeta 2 glicoproteína I y el anticoagulante lúpico mientras que otros han relacionado los anticuerpos anticardiolipina $\operatorname{IgG}$ o IgA, este último en IAM con elevación del ST. Se han reportado casos fatales en pacientes jóvenes, donde las manifestaciones cardiacas ocurren por desórdenes inmunes y por su asociación con fenómenos trombóticos (25-30).

Se piensa que luego de un IAM en pacientes con SAF, $15 \%$ producen nuevos anticuerpos, en contraste con el $4 \%$ de la población sin la enfermedad; la persistencia de los anticuerpos en sangre aumenta la posibilidad de recurrencia y se sabe que, en hombres sanos, la presencia de anticardiolipina positivo aumenta dos veces el riesgo de un evento coronario. La etiopatogenia podría asociarse a la presencia de los anticuerpos, a la injuria vascular y a la exposición de neo antígenos. En nuestro país encontramos un caso reportado en la literatura de un paciente joven de 24 años (31).

\section{CONCLUSIONES}

El IAM típicamente se presenta en pacientes mayores que tienen factores de riesgo cardiovascular como hipertensión y dislipidemia (20), siendo una manifestación rara de SAF pero que, en pacientes jóvenes sin factores de riesgo, debe sospecharse y tratarse a tiempo, teniendo en cuenta que, requiere del manejo interdisciplinario de cardiólogos, reumatólogos, intensivistas e incluso, médicos intervencionistas, debido a que impacta en la morbimortalidad de los pacientes con SAF primario. Por ello, es fundamental iniciar el manejo para integral cubriendo el IAM y la enfermedad de base, así como es importante educar al paciente sobre la enfermedad haciendo hincapié en la adherencia al tratamiento y modificación de factores de riesgo para mantener el riesgo en el menor limite posible.

Conflicto de Interés: Los autores declaran NO tener ningún conflicto de interés.

\section{REFERENCIAS}

1. Negrini S, Pappalardo F, Murdaca G, et al. The antiphospholipid syndrome: from pathophysiology to treatment. Clin Exp Med. 2017;17(3):257-67.

2. Clinical manifestations of antiphospholipid syndrome - UpToDate [Internet]. [citado 21 de octubre de 2019]. Disponible en: https://wwwuptodate-

com.recursosenlinea.juanncorpas.edu.co:2443/contents/clinicalmanifestations-of-antiphospholipid-

syndrome? search=Clinical $\% 20$ manifestations $\% 20$ of $\% 20$ antiphospholipi $\mathrm{d} \% 20$ syndrome\&source $=$ search_result\&selectedTitle $=1 \sim 150 \&$ usage_ty pe $=$ default\&display_rank $=1$

3. Matyja-Bednarczyk A, Swadźba J, Iwaniec T, et al. Risk factors for arterial thrombosis in antiphospholipid syndrome. Thromb Res. 2014;133(2):173-6.

4. Nazir S, Tachamo N, Lohani S, et al Acute myocardial infarction and antiphospholipid antibody syndrome: a systematic review. Coron Artery Dis.2017;28(4):332-5.

5. Davies JOJ, Hunt BJ. Myocardial infarction in young patients without coronary atherosclerosis: assume primary antiphospholipid syndrome until proved otherwise. Int J Clin Pract. 2007;61(3):379-84.

6. Osula S, Bell GM, Hornung RS. Acute myocardial infarction in young adults: causes and management. Postgrad Med J. 2002;78(915):27-30. 
7. Kolitz T, Shiber S, Sharabi I, et al. Cardiac Manifestations of Antiphospholipid Syndrome With Focus on Its Primary Form. Front Immunol. 2019;10:941.

8. Cervera R, Piette J-C, Font J, et al. Antiphospholipid syndrome: clinical and immunologic manifestations and patterns of disease expression in a cohort of 1,000 patients. Arthritis Rheum.2002;46(4):1019-27.

9. Prashanth $\mathrm{P}$, Mukhaini M, Riyami A. A rare presentation of primary antiphospholipid syndrome. Oman Med J. 2009;24(4):300-2 .

10. Hughes G. Hughes syndrome (antiphospholipid syndrome) and myocardial infarction. Int J Clin Pract. 2010;64(9):1183-4.

11. Gandhi H, Ahmed N, Spevack DM. Prevalence of myocardial infarction with non-obstructive coronary arteries (MINOCA) amongst acute coronary syndrome in patients with antiphospholipid syndrome. Int $\mathrm{J}$ Cardiol Heart Vasc. 2019;22:148-9.

12. Takeuchi S, Obayashi T, Toyama J. Primary antiphospholipid syndrome with acute myocardial infarction recanalised by PTCA. Heart Br Card Soc. 1998;79(1):96-8.

13. Grosso G, Sippl N, Kjellstrom B, et al. Antiphospholipid Antibodies in Patients With Myocardial Infarction. Ann Intern Med. 2019;170(4):27780.

14. Gannon S, Hyder O. Treatment of ST elevation Myoccardial Infarction in Antiphospholipid Syndrome J Am Coll Cardiol. 2018;71(11 Supplement):A2196.

15. Erkan D, Lockshin MD. Antiphospholipid Syndrome. First. New York: Springer; 2017.

16. Marti V, Seixo F, Santalo M, et al. Antiphospholipid syndrome and acute myocardial infarction: treatment with thrombectomy and abciximab. Rev Port Cardiol Orgao Of Soc Port Cardiol Port J Cardiol Off J Port Soc Cardiol. 2014;33(7-8):465.e1-4.

17. George D, Erkan D. Antiphospholipid syndrome. Prog Cardiovasc Dis. 2009;52(2):115-25.

18. Cervera R. Antiphospholipid syndrome. Thromb Res. 2017;151:S43-7.

19. Stepien K, Nowak K, Wypasek E, et al. High prevalence of inherited thrombophilia and antiphospholipid syndrome in myocardial infarction with non-obstructive coronary arteries: Comparison with cryptogenic stroke. Int J Cardiol. 2019;290:1-6.

20. Perl L, Netzer A, Rechavia E, et al. Long-term outcome of patients with antiphospholipid syndrome who undergo percutaneous coronary intervention. Cardiology. 2012;122(2):76-82.

21. Tektonidou MG, Andreoli L, Limper M, et al. EULAR recommendations for the management of antiphospholipid syndrome in adults. Ann Rheum Dis. 2019;78(10):1296-304.

22. Negrini S, Pappalardo F, Murdaca G, et al. The antiphospholipid syndrome: from pathophysiology to treatment. Clin Exp Med. 2017;17(3):257-67.

23. Koniari I, Siminelakis SN, Baikoussis NG, et al. Antiphospholipid syndrome; its implication in cardiovascular diseases: a review. J Cardiothorac Surg. 2010;5:101.

24. Smukowska-Gorynia A, Mularek-Kubzdela T, Araszkiewicz A. Recurrent acute myocardial infarction as an initial manifestation of antiphospholipid syndrome: treatment and management. Blood Coagul Fibrinolysis Int J Haemost Thromb. 2015;26(1):91-4.

25. Abid L, Frikha F, Bahloul Z, et al. Acute myocardial infarction in young adults with antiphospholipid syndrome: report of two cases and literature review. Pan Afr Med J. 2011;8:13.

26. Hughes GR. The antiphospholipid syndrome: ten years on. Lancet Lond Engl. 1993;342(8867):341-4.

27. Dhibar DP, Sahu KK, Varma SC, et al. Intra-cardiac thrombus in antiphospholipid antibody syndrome: An unusual cause of fever of unknown origin with review of literature. J Cardiol Cases. 2016;14(5):153-6.

28. Waisayarat J, Plumworasawat S, Vilaiyuk S, et al. Intracardiac thrombus in a patient with catastrophic antiphospholipid syndrome: an autopsy case report and review of the literature. Vasc Health Risk Manag. 2019;15:253-8

29. Cakmak HA, Aslan S, Durmaz E, et al. Acute myocardial infarction due to antiphospholipid antibody syndrome in a young pregnant woman. J Cardiol Cases. 2011;4(1):e8-12.

30. Sharma AK, Baig MW, Heist EK. Intracardiac thrombosis and acute myocardial infarction as initial presentation of antiphospholipid syndrome. Am J Med Sci. 2011;342(3):254-6.

31. Uribe CE, Cárdenas JM, Cabrales J, et al. Infarto agudo del miocardio como primera manifestación del síndrome antifosfolípido primario en un paciente de veinticuatro años. Rev Colomb Cardiol. 2005;12(3):5.

32. 